\title{
Educación integral de la sexualidad en la formación de estudiantes de psicología
}

\author{
Integral sex education in the training of psychology students
}

\author{
Claudia Salinas Boldo \\ Facultad de Ciencias Humanas de la Universidad Autónoma de Baja California \\ claudia.salinas.boldo@uabc.edu.mx \\ https://orcid.org/0000-0003-4207-6970 \\ Pedro Antonio Be Ramírez \\ Facultad de Ciencias Humanas de la Universidad Autónoma de Baja California \\ https://orcid.org/0000-0002-9798-9366
}

\section{Resumen}

El objetivo de este trabajo fue el de describir y analizar el contenido y aplicación del programa de la unidad académica (PUA) de "Sexualidad humana" del programa de licenciatura en psicología, de la Universidad Autónoma de Baja California (UABC). Esto en función de la vinculación que debe existir entre dicha formación y tanto los lineamientos internacionales en materia de educación sexual y desarrollo sostenible, como los ejes transversales de equidad y responsabilidad social universitaria del Plan de Desarrollo Institucional (PDI) de la UABC 2019-2023. Se aplicaron las técnicas de mapeo con base en los programas educativos de 32 universidades públicas del país, análisis de contenido al PUA de "Sexualidad humana" y tres grupos focales con nueve estudiantes que ya habían cursado la UA anteriormente mencionada. Se encontró que más de la mitad de las universidades públicas del país integran materias vinculadas con la sexualidad dentro de sus programas de licenciatura en psicología; que el PUA analizado requiere de la inclusión de temas vinculados con el aspecto sociocultural de la sexualidad, tales como: valores y sexualidad; cultura y sexualidad; derechos sexuales y reproductivos; violencia de género; inclusión y diversidad sexual y estigma e ITS., y que los y las estudiantes, reconocen que los y las psicólogas tendrían que estar preparados para llevar a cabo procesos de educación sexual integral, algo que reconocen como una necesidad urgente en la sociedad mexicana.

Palabras clave: educación sexual; educación superior; sexualidad; jóvenes 


\begin{abstract}
The objective of this research was to describe and analyze the content and application of the academic unit program (PUA) of "Human Sexuality" of the degree program in psychology at the Autonomous University of Baja California (UABC). This analysis based on the link that must exist between such training and both the international guidelines on sexual education and sustainable development, as well as the transversal axes of equity and university social responsibility of the Institutional Development Plan (PDI) of the UABC 2019- 2023. The techniques used were: mapping based on the educational programs of 32 public universities in the country; content analysis to the PUA of "Human Sexuality" and three focus groups with nine students who had already completed the aforementioned UA. It was found that more than half of the country's public universities integrate subjects related to sexuality into their psychology degree programs; that the analyzed PUA requires the inclusion of topics related to the socio-cultural aspect of sexuality, such as: values and sexuality; culture and sexuality; sexual and reproductive rights; gender violence; inclusion and sexual diversity and stigma and STIs, and that students recognize that psychologists should be prepared to carry out comprehensive sexual education processes, something that they recognize as an urgent need in Mexican society.
\end{abstract}

Key words: sex education; higher education; sexuality; youth 


\section{Introducción}

La sexualidad es una de las dimensiones que nos conforman como seres humanos. La salud sexual es parte de nuestra salud integral y la educación integral de la sexualidad, es el medio que nos permitirá alcanzar el objetivo de lograr una salud sexual completa, en los aspectos biológico, social y psicológico. Al ser la psicología la disciplina enfocada en la conducta y el bienestar emocional del ser humano, sería deseable que quien se encuentre en proceso de formación en esta área del conocimiento, reciba educación integral de la sexualidad, para estar en posibilidad de promover la salud sexual integral de las personas durante su vida profesional

Un o una profesional de la psicología que no sepa cómo abordar la sexualidad humana como fenómeno biológico, psicológico, emocional, social, cultural e incluso político, será un profesional que cuente con menos herramientas para comprender y dar respuesta a las problemáticas, tanto individuales como sociales, que se deriven del aspecto sexual de los seres humanos. (Álvarez-Gayou, 1998)

La UABC cuenta con una unidad de aprendizaje (UA) obligatoria, destinada al estudio de la sexualidad humana, en el programa de licenciatura en psicología que opera desde el 2012. A 7.5 años -15 semestres- de la implementación del programa de la unidad de aprendizaje (PUA) de "Sexualidad humana", nos parece necesario el llevar a cabo un primer acercamiento exploratorio, que nos permita identificar aciertos y áreas de oportunidad tanto del PUA como de su aplicación, con el objetivo de diseñar e implementar mejores estrategias educativas para el beneficio de nuestra comunidad estudiantil y docente.

El objetivo de esta investigación es el de describir y analizar el contenido y aplicación del PUA de "Sexualidad humana" del programa de licenciatura en psicología que se imparte en dos unidades académicas de la UABC. Lo anterior en función de la vinculación que debe existir entre dicha formación y tanto los lineamientos internacionales en materia de educación sexual y desarrollo sostenible, como los ejes transversales de equidad y responsabilidad social universitaria del Plan de Desarrollo Institucional (PDI) de la UABC 2019-2023.

\section{Marco teórico}

La Organización de las Naciones Unidas para la Educación, la Ciencia y la Cultura (UNESCO, 2014: 33), indica que la sexualidad "nos define como personas, forma parte de nuestro desarrollo y expresión a lo largo de nuestro ciclo vital..." y agrega que la vulneración de la sexualidad "...afecta nuestra salud y nuestro bienestar, [la sexualidad] tiene directa relación con el cuidado y [...] con la forma como nos relacionamos con otras personas"

A pesar de que la sexualidad es una de las dimensiones que nos constituyen como seres humanos y por ende, como sociedad, es común que ésta sea tratada desde sus aspectos exclusivamente médico-biológicos y abordada desde la heteronormatividad, lo cual contribuye a perpetuar la violencia de género, la exclusión a las sexualidades no heterosexuales y los estereotipos. (ÁlvarezGayou, 1998; UNESCO, 2014 y Rosales-Mendoza y Salinas-Quiroz, 2017)

Una de las disciplinas desde las cuales tendría que abordarse la sexualidad es la psicología, ya que son los y las profesionales de esta área del conocimiento -junto con educólogos, trabajadores sociales, sociólogos, entre otros-, a quienes correspondería tanto diseñar, como implementar y acompañar, procesos de educación sexual integral en los diferentes espacios en los cuales se necesiten. Cabe mencionar que si bien la sexología es la disciplina a la que, directamente, deberían asignársele estas tareas, no existe -al menos en México- aún un programa de licenciatura en 
sexología. Los y las sexólogas de México, cuentan con diversas licenciaturas, ya que la formación sexológica, solamente se encuentra disponible en forma de especialidad, maestría o doctorado.

A pesar de lo anteriormente mencionado, no todos los programas de licenciatura en psicología cuentan con asignaturas -ya sea en la modalidad de obligatorias u optativas-, destinadas al estudio integral de la sexualidad humana. La Universidad Autónoma de Baja California (UABC) es una de las instituciones que incluye dentro de su programa de licenciatura en psicología, la asignatura o UA de "Sexualidad humana" en modalidad obligatoria. Esta asignatura se imparte en tres unidades académicas distintas de la UABC, durante sexto semestre, en la etapa disciplinar de la licenciatura.

La UNESCO (2014) considera la educación de la sexualidad como una competencia básica, pues implica hablar de manera abierta acerca de temas tales como la diversidad sexual, el género y el placer, desde una perspectiva integral, que involucre tanto nuestra dimensión biológica como la afectiva y la social. Brindar educación sexual, requiere de una formación que los profesores y las profesoras no siempre tienen, así como una ética profesional basada en valores como el respeto, la inclusión y la responsabilidad.

Este estudio pretende llevar a cabo un análisis del proceso de formación en sexualidad que se brinda en la asignatura "Sexualidad humana", del programa de licenciatura en psicología de la UABC, desde sus contenidos y desde la percepción de los y las estudiantes que han cursado esa UA. Lo anterior con la finalidad de conocer el impacto que este espacio de formación ha tenido, así como su pertinencia, con respecto a los lineamientos internacionales en materia de educación sexual dictados por la UNESCO (2018), los objetivos desarrollo sostenible dados por la ONU (2015) y los ejes transversales de equidad y responsabilidad social universitaria del PDI-UABC 2019-2023.

Un estudio de objetivo similar al que se presenta en este documento, es el de González (2015), quien llevó a cabo un estudio de corte cualitativo, con 53 estudiantes colombianos -14 varones y 39 mujeres- de seis programas de licenciatura en psicología distintos. El objetivo de dicha investigación fue el de explorar la concepción que los participantes tienen acerca de la sexualidad. La autora encontró que la sexualidad no se contempla en el currículo como asignatura obligatoria u optativa. La sexualidad se menciona en la licenciatura, vinculada a la psicopatología o en sus aspectos biológicos. Se detecta la necesidad de trabajar la sexualidad, vinculada a la dimensión afectiva y social, así como incluir la perspectiva de género y el respeto a la diversidad sexual.

En 2009, Chávez, Petrzelová y Zapata, llevaron a cabo un estudio con el objetivo de identificar los conocimientos, actitudes y prácticas respecto a la sexualidad en 719 jóvenes estudiantes de nivel superior mexicanos. Las autoras y el autor encontraron que los y las jóvenes han tenido una educación sexual deficiente o "nula" y que la escuela no ha tenido un papel significativo como institución formadora en torno a este tema. Los y las jóvenes carecían de información acerca de anatomía, género, infecciones de transmisión sexual y derechos sexuales y reproductivos, entre otros. Asimismo, se encontraron muchos prejuicios y actitudes de rechazo hacia las preferencias no heterosexuales, así como una baja percepción de riesgo hacia las infecciones de transmisión sexual. Lo anterior en el contexto de actitudes basadas en valores tradicionales y conocimientos populares acerca de la sexualidad.

También en México y en 2009, Moral y Ortega, llevaron a cabo un estudio con 395 estudiantes de psicología, con el objetivo de analizar tanto las representaciones sociales como las actitudes de estos y estas estudiantes hacia la sexualidad. Los resultados apuntan a actitudes de mayor apertura y aceptación hacia diversas manifestaciones de la sexualidad ya que, si bien se encontró que los y las jóvenes valoran la virginidad, a la vez, aceptan la masturbación y la homosexualidad. Asimismo, el respeto y la higiene parecen ser elementos vinculados de manera significativa al concepto de sexualidad. 
Cañizo y Salinas (2010), hicieron una investigación con 851 estudiantes de nivel superior en México, con el objetivo de conocer la relación existente entre permisividad y conductas sexuales alternas y describir el tipo de prácticas realizadas por los y las jóvenes universitarias. Como parte de los resultados de este estudio, se reportó que los y las estudiantes de psicología, reportaron menos conductas sexuales alternas, algo que la autora y el autor atribuyen a la mayor presencia de población femenina en la licenciatura en psicología, por la represión que caracteriza la educación sexual de las mujeres en un país sexista como lo es México. Además, consideran que es posible que estas estudiantes hayan respondido con una mayor reserva, ante el temor de poner en duda sus niveles de salud mental, pues las conductas sexuales alternas, es decir, aquellas que se alejan del coito heterosexual, aún son consideradas como expresiones de desequilibrio mental.

Resultados similares se obtuvieron en un estudio llevado a cabo por Hernández et al. (2017) con 122 estudiantes ecuatorianos de la licenciatura en psicología clínica. Se encontró que los y las estudiantes se encuentran desinformados en torno a temas vinculados con la salud sexual como el uso de métodos anticonceptivos y las infecciones de transmisión sexual. Asimismo, se detectaron actitudes de rechazo hacia la homosexualidad, así como una relación entre el inicio de la vida sexual activa y el consumo de alcohol y otras sustancias.

En 2017, el Consejo Nacional de Población (CONAPO), dio a conocer que la principal necesidad en materia de salud sexual y reproductiva, en Baja California es el acceso a información, educación, orientación y servicios en salud sexual y reproductiva, pues la mitad de las mujeres que inicia su vida sexual lo hace de manera desprotegida. Lo anterior ha traído como consecuencia un descenso en la edad en la que las mujeres tienen a su primer hijo o hija, lo cual nos lleva al fenómeno del embarazo adolescente que está profundamente vinculado con la necesidad de servicios tanto educativos como sanitarios, en materia de salud sexual y reproductiva. La brecha entre la edad mediana en la que inician las relaciones sexuales y la edad mediana en la que se inicia el uso de algún método anticonceptivo es amplia, lo cual deja a las mujeres desprotegidas, por un período considerable de tiempo. Cabe mencionar que en este estudio se toma en cuenta a mujeres en un rango de edad que va de los 15 a los 49. Sin embargo, se menciona la necesidad de que los hombres incrementen su participación en el uso de métodos anticonceptivos, ya que es necesario implementar la doble protección en la vida sexual, de tal manera que se eviten tanto los embarazos no deseados como las infecciones de transmisión sexual.

De acuerdo con la más reciente Encuesta Nacional sobre la Dinámica de las Relaciones en los Hogares (ENDIREH), llevada a cabo por el Instituto Nacional de Estadística y Geografía (INEGI) en 2016, en México, el 66.1\% de las mujeres mayores de 15 años, han sufrido al menos un incidente de violencia a lo largo de su vida. De ellas, el $49 \%$ han sido víctimas de violencia emocional y el $41.3 \%$, de violencia sexual, que son los dos tipos de violencia que más reportan las mujeres mexicanas. Este mismo estudio indica que el $66.2 \%$ de las mujeres en Baja California han experimentado algún tipo de violencia. El 23.2\% en el ámbito escolar, el 32.2\% en el ámbito laboral, el $37.7 \%$ en la comunidad y el $35 \%$ ha sido agredida por su pareja.

De acuerdo con la Encuesta Nacional sobre Discriminación en México (ENADIS) realizada por el Consejo Nacional para Prevenir la Discriminación en México (CONAPRED) en 2010, más de la mitad de la población se encuentra en desacuerdo con que las parejas homosexuales adopten a menores, 4 de cada 10 mexicanos y mexicanas no estarían dispuestos a permitir que en su casa vivieran personas homosexuales y 3 de cada 10 no estarían dispuestos a vivir en la misma casa con una persona seropositiva. Por otro lado, la mitad de las personas que se identifican como homosexuales, lesbianas o bisexuales, consideran que el principal problema al que se enfrentan en México, es la discriminación, seguida de la falta de aceptación y 7 de cada 10 personas de esta misma población, considera que en México, no se respetan los derechos de la comunidad LGBT+ 
Los datos anteriormente mencionados, coinciden con lo reportado por parte de la Organización para la Cooperación y el Desarrollo Económico (OCDE, 2020), que califica el nivel de inclusión actual de la comunidad LGBT+ en México, como "moderado", pues en una escala que va del 1 al 10, tiene un nivel de inclusión de 5. Por otro lado, este mismo organismo, reportó que de los 33 países que lo integran, México ocupa el primer lugar en materia de abuso sexual infantil (Senado de la República, 2019)

Estos datos nos brindan un panorama contundente acerca de la urgencia de brindar a nuestros y nuestras profesionales de la psicología, elementos que les permitan abordar las problemáticas vinculadas con la sexualidad humana, no solamente desde lo individual-familiar, sino también desde lo colectivo-social, vinculando estas intervenciones con objetivos políticos tales como los de la inclusión social, la equidad y la cultura de la paz. Lo anterior resulta particularmente significativo cuando hablamos de estudiantes de instituciones públicas, pues los objetivos y acciones de la universidad pública, tendrían que estar orientados a dar respuesta a las demandas y problemáticas sociales más urgentes. (UABC, 2019)

\section{Metodología}

Esta investigación se llevó a cabo desde un enfoque cualitativo, aplicando las siguientes técnicas:

a) Mapeo basado en una exploración de páginas oficiales de universidades públicas de las 32 entidades federativas de la república mexicana. Una universidad pública por entidad. En el caso de la Ciudad de México, se eligió el caso de la Universidad Nacional Autónoma de México (UNAM). En este mapeo, se buscó responder a las preguntas de: ¿Cuáles, de las universidades públicas del país que ofrecen la licenciatura en psicología, incluyen materias vinculadas al tema de la sexualidad en sus planes de estudio? y ¿Cuáles de estas universidades consideran estas materias como obligatorias? Esto con el objetivo de conocer la importancia que estas instituciones dan al tema de la sexualidad, en la formación de los y las psicólogas.

b) Análisis de contenido del PUA de "Sexualidad humana", con base en a) Los objetivos de desarrollo sostenible dados por la ONU (2015), b) Las Orientaciones técnicas internacionales sobre educación en sexualidad de la UNESCO (2018) y c) Los ejes transversales de responsabilidad social universitaria y equidad, enunciados en el PDI-UABC 2019-2023.

c) Grupo focal. Tres grupos focales, integrados por nueve estudiantes en total: dos hombres y siete mujeres, de 22 a 25 años de edad, que ya han cursado la UA de "Sexualidad humana". Estos grupos focales fueron aplicados por tres estudiantes -mujeres- pares de los y las estudiantes participantes. Lo anterior se decidió, para minimizar el sesgo de la deseabilidad social en las respuestas de los y las participantes ya que, la autora y el autor de este texto, pertenecemos al grupo de docentes de la licenciatura en psicología. Estos grupos focales fueron audiograbados y los análisis se hicieron sobre las transcripciones. A los y las participantes se les asignó un seudónimo, para poder mostrar extractos textuales de sus respuestas en este documento. Los y las estudiantes que participaron en los grupos focales, dieron su consentimiento como parte de la audiograbación. 


\section{Resultados}

\subsection{Mapeo}

Se incluyeron en la muestra las universidades públicas de las 32 entidades federativas de la república mexicana. De estas universidades, 3 no cuentan con la licenciatura en psicología y de las demás no se encontró suficiente información en su página oficial. En la tabla se muestran las 19 universidades cuya información se encuentra en línea, que cuentan con la licenciatura en psicología y que incluyen una o más materias, asignaturas o unidades de aprendizaje vinculadas con el tema de la sexualidad, en el plan de estudios de dicha licenciatura. En la tabla también se indica el semestre o nivel en el que se imparte esta asignatura. La palabra "Flexible" se escribió en aquellas instituciones que permiten que los y las estudiantes cursen la asignatura en cualquier semestre. En la última columna se indica si esta asignatura es considerada como obligatoria o no.

Tabla 1

Resultado del mapeo

\begin{tabular}{|c|c|c|c|}
\hline Universidad & Nombre & Semestre & Obligatoria \\
\hline 1.- Universidad Autónoma de Baja California & Sexualidad humana & 6 & Sí \\
\hline 2.- Universidad Autónoma de Yucatán & Sexualidad humana & Flexible & Sí \\
\hline \multirow[t]{2}{*}{ 3.- Universidad de Guanajuato } & a) Sexualidad e identidad de género & 7 & No \\
\hline & $\begin{array}{l}\text { b) Sexualidad y paternidad } \\
\text { responsable }\end{array}$ & & \\
\hline 4.- Universidad de Colima & Sexualidad y género & 4 & Sí \\
\hline 5.- Universidad Autónoma de Campeche & Sexualidad & 6 & Sí \\
\hline 6.- Universidad Autónoma del Estado de Hidalgo & Teoría de género y sexualidad & 6 & Sí \\
\hline $\begin{array}{l}\text { 7.- Universidad Michoacana de San Nicolás de } \\
\text { Hidalgo }\end{array}$ & Sexualidad y género & 4 & Sí \\
\hline 8.- Universidad Autónoma de Nuevo León & Sexualidad humana & 7 & Sí \\
\hline 9.- Universidad Autónoma “Benito Juárez” de Oaxaca & Sexualidad humana & 5 & Sí \\
\hline 10.- Benemérita Universidad Autónoma de Puebla & Sexualidad & Básico & Sí \\
\hline 11.- Universidad Autónoma de Querétaro & Educación de la sexualidad & 1 & Sí \\
\hline 12.- Universidad Autónoma de San Luis Potosí & $\begin{array}{l}\text { a) Coeducación y diferencia sexual } \\
\text { b) Salud sexual y afectiva }\end{array}$ & Flexible & No \\
\hline 13.- Universidad Autónoma de Sinaloa & Sexualidad humana & 4 & Sí \\
\hline 14.- Universidad de Sonora & $\begin{array}{l}\text { a) Educación en sexualidad humana } \\
\text { b) Salud sexual y reproductiva }\end{array}$ & Flexible & No \\
\hline 15.- Universidad Juárez Autónoma de Tabasco & $\begin{array}{l}\text { Estudios de género y prácticas } \\
\text { contemporáneas de la sexualidad }\end{array}$ & $\begin{array}{c}\text { Área sustantiva } \\
\text { profesional }\end{array}$ & Sí \\
\hline 16.- Universidad Autónoma de Coahuila & Sexualidad humana & 2 & Sí \\
\hline 17.- Universidad Autónoma de Tamaulipas & Sexualidad humana & 7 & Sí \\
\hline 18.- Universidad Nacional Autónoma de México & Sexualidad humana y género & 8 & No \\
\hline 19.- Universidad de Guadalajara & Sexualidad humana & $\begin{array}{c}\text { Área de } \\
\text { formación } \\
\text { básica común }\end{array}$ & Sí \\
\hline
\end{tabular}

Fuente: Elaboración propia 


\subsection{Contenido del PUA}

La UA de "Sexualidad humana", tiene como propósito general el contribuir a la formación de profesionales de la Psicología, en la comprensión de los fundamentos teórico-prácticos del ejercicio de la sexualidad humana, desde una perspectiva integral, así como de los procesos que caracterizan a los aspectos relativos a la salud sexual, como fundamento para contribuir a la mejora de la calidad de vida, a través de la difusión de programas educativos en materia de orientación sexual, destacando los aspectos éticos y sociales que norman a la práctica de la sexualidad (UABC, 2012)

En la tabla 1, se realizó un ejercicio comparativo entre los contenidos del PUA de "Sexualidad humana" de la UABC, los contenidos clave en educación sexual (CCES) de la UNESCO y los objetivos de desarrollo sostenible (ODS) de la ONU. Se colocaron en el mismo nivel los elementos que se vinculan entre sí. LOS CCES-UNESCO y los ODS-ONU a los que no se les encontraron elementos similares en el PUA-UABC, quedaron fuera de esta tabla.

Tabla 2

Comparativa entre PUA-UABC, CCES-UNESCO y ODS-ONU

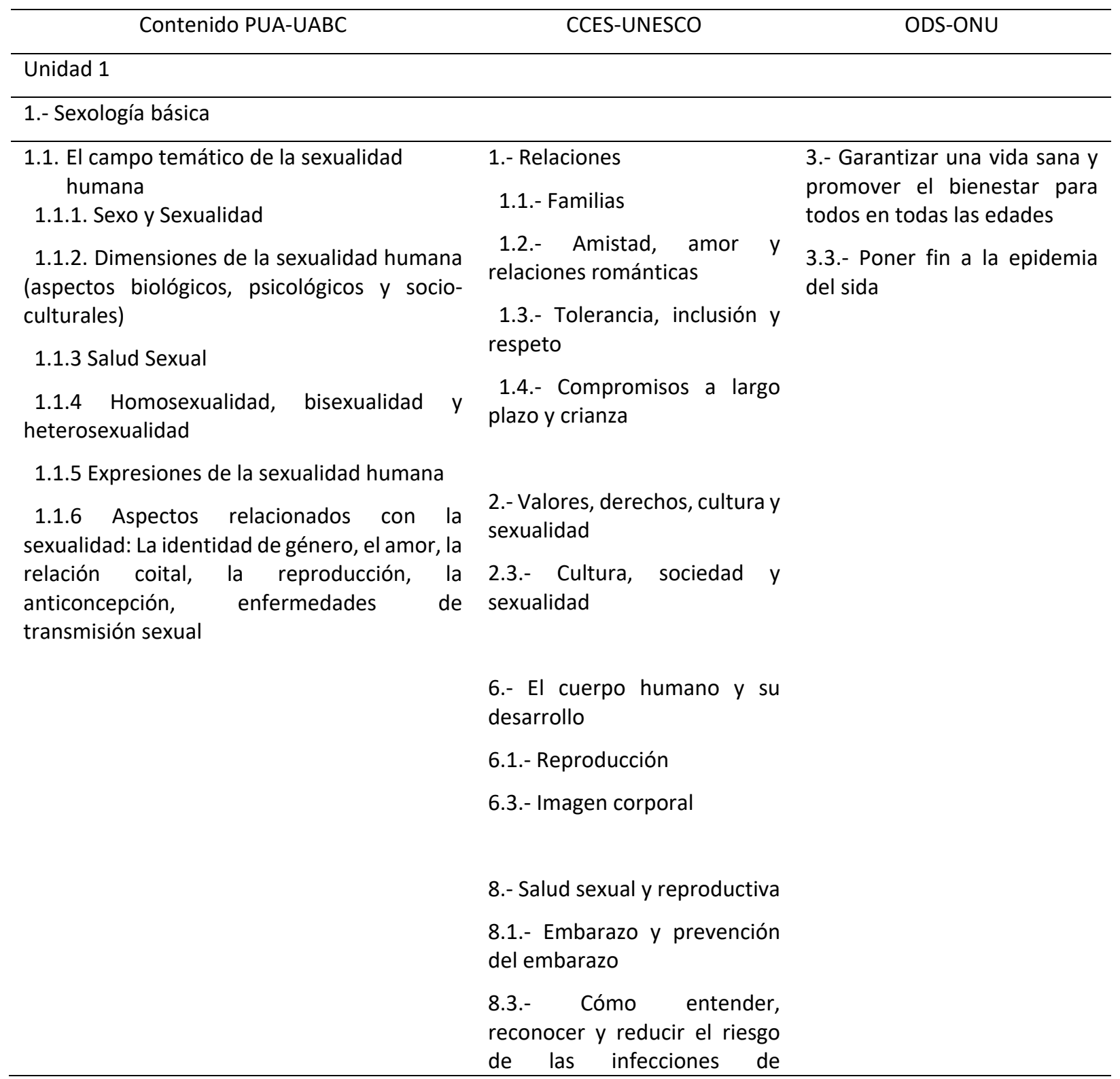

Revista del Laboratorio Iberoamericano para el Estudio Sociohistórico de las Sexualidades https://doi.org/10.46661/relies.5162 
transmisión sexual, incluido el

1.2.- Desarrollo Psicosexual

\subsection{Adolescencia y Sexualidad}

1.3.1 Cambios físicos, psicológicos y sexuales

1.3.2 Manejo adolescente de la sexualidad
6.- El cuerpo humano y su

desarrollo

6.2.- Pubertad

6.3.- Imagen corporal

7.- Sexualidad y conducta

sexual

7.1.- Relaciones sexuales,

y sexualidad y ciclo de vida

sexual

7.2.- Conducta sexual y

respuesta sexual

1.5 Embarazo y sexualidad

1.5.1 Respuesta sexual

1.5.2 Erotismo y desempeño sexual

1.5.3 Actitudes psicológicas de la mujer embarazada

1.5.4 Reacciones masculinas frente al embarazo

1.5.5 La sexualidad en el climaterio femenino y masculino

1.5.6 Respuesta sexual y perturbaciones de la sexualidad

Unidad 2

2. Disfunciones sexuales

2.1 Respuesta sexual y disfunciones

2.2 Etiología de las disfunciones sexuales

2.3 Diagnóstico de las disfunciones sexuales

2.4 Métodos y técnicas de intervención para el tratamiento de las disfunciones sexuales

2.5 Fases para la atención de la salud sexual

2.6 Esquema de atención para no especialistas

2.7 Aspectos éticos y sociales del tratamiento de las disfunciones sexuales

Fuentes: UABC (2012), ONU (2015) y UNESCO (2018)

La bibliografía se compone de 11 libros y en ese mismo espacio, se recomienda la película "Éxtasis".

Revista del Laboratorio Iberoamericano para el Estudio Sociohistórico de las Sexualidades https://doi.org/10.46661/relies.5162 
En la tabla 2 se llevó a cabo una síntesis de los contenidos clave en educación sexual (CCES) de la UNESCO. Los contenidos que se encuentran en color gris, son los que no cuentan con elementos similares en el PUA-UABC de "Sexualidad humana"

Tabla 3

CCES-UNESCO no incluidos en PUA-UABC

\begin{tabular}{|c|c|}
\hline 1.- Relaciones & 5.- Habilidades para la salud y el bienestar \\
\hline 1.1.- Familias & 5.1.- Influencia de normas y grupos de pares en la \\
\hline 1.2.- Amistad, amor y relaciones románticas & sexu \\
\hline 1.3.- Tolerancia, inclusión y respeto & 5.2.- Toma de decisiones \\
\hline \multirow[t]{3}{*}{ 1.4.- Compromisos a largo plazo y crianza } & $\begin{array}{l}\text { 5.3.- Habilidades de comunicación, rechazo y } \\
\text { negociación }\end{array}$ \\
\hline & 5.4.- Alfabetización mediática y sexualidad \\
\hline & 5.5.- Cómo encontrar ayuda y apoyo \\
\hline
\end{tabular}

2.- Valores, derechos, cultura y sexualidad

6.- El cuerpo humano y su desarrollo

2.1.- Valores y sexualidad

6.1.- Reproducción

2.2.- Derechos humanos y sexualidad

6.2.- Pubertad

2.3.- Cultura, sociedad y sexualidad

6.3.- Imagen corporal

3.- Género

3.1.- Construcción social del género y de las normas de género

3.2.- Igualdad, estereotipos y prejuicios de género

3.3.- Violencia de género
7.- Sexualidad y conducta sexual

7.1.- Relaciones sexuales, sexualidad y ciclo de vida sexual

7.2.- Conducta sexual y respuesta sexual
4.- Violencia y seguridad personal

\section{1.- Violencia}

4.2.- Consentimiento, privacidad e integridad física

4.3.- Uso seguro de tecnologías de información y comunicación (TIC)
8.- Salud sexual y reproductiva

8.1.- Embarazo y prevención del embarazo

8.2.- Estigma del VIH y del sida, atención médica, tratamiento y apoyo

8.3.- Cómo entender, reconocer y reducir el riesgo de las infecciones de transmisión sexual, incluido el VIH

Fuente: UNESCO (2018)

En la tabla 3 se describen los objetivos de desarrollo sostenible de la OMS, vinculados con el tema de la sexualidad. Los contenidos que se encuentran en color gris, son los que no cuentan con elementos similares en el PUA-UABC de "Sexualidad humana"

Tabla 4

ODS-OMS no incluidos en PUA-UABC

\begin{tabular}{ll}
\hline 3.- Garantizar una vida sana y & 3.3.- Poner fin a la epidemia del sida \\
promover el bienestar para & 3.7.- Garantizar el acceso universal a los servicios de salud sexual y \\
todos en todas las edades & $\begin{array}{l}\text { reproductiva, incluidos los de planificación de la familia, información y } \\
\text { educación, y la integración de la salud reproductiva }\end{array}$
\end{tabular}

Revista del Laboratorio Iberoamericano para el Estudio Sociohistórico de las Sexualidades https://doi.org/10.46661/relies.5162 
5.- Lograr la igualdad entre los géneros y empoderar a todas las mujeres y las niñas
5.1.- Poner fin a todas las formas de discriminación contra todas las mujeres y las niñas en todo el mundo

5.2.- Eliminar todas las formas de violencia contra todas las mujeres y las niñas en el ámbito público y privado, incluidas la trata y la explotación sexual y otros tipos de explotación

$\begin{array}{ll}\begin{array}{l}\text { 10.- Reducción de las } \\ \text { desigualdades }\end{array} & \begin{array}{l}\text { 10.2.- Potenciar y promover la inclusión social, económica y política de } \\ \text { todas las personas, independientemente de su edad, sexo, discapacidad, } \\ \text { raza, etnia, origen, religión o situación económica u otra condición }\end{array}\end{array}$

Fuente: ONU (2015)

Las metas de los ODS que no se encuentran incluidas en el PUA de "Sexualidad humana", se encuentran significativamente vinculadas con los ejes tranversales de equidad y responsabilidad social universitaria del PDI-UABC, cuya descripción podemos encontrar en la tabla 5.

Tabla 5

Ejes transversales del PDI-UABC 2019-2023

\begin{tabular}{ll}
\hline Responsabilidad & “...compromiso de cooperación con los distintos sectores y grupos sociales, en \\
social & particular con los que presentan mayor desventaja [...] formación integral de \\
universitaria & los estudiantes, a través del ejercicio responsable de la ciudadanía, la \\
& promoción de una cultura de la paz, así como de los derechos humanos [...] \\
& producción de conocimientos que contribuyan a revertir la desigualdad y \\
& atiendan las necesidades del entorno" (UABC, 2019: 95)
\end{tabular}

Equidad “...partimos del respeto y reconocimiento a la igualdad de género y a la
diversidad en todas sus expresiones socioculturales y políticas..." (UABC, 2019:
$96)$

Fuente: (UABC, 2019)

\subsection{Grupos focales de estudiantes}

En los grupos focales, siete participantes indicaron que les parecía adecuado que la UA fuera obligatoria en la licenciatura en psicología y que también estuviera disponible en otras licenciaturas, dado que hay mucho desconocimiento en torno al tema, pues en niveles académicos anteriores, no se brinda una formación en sexualidad adecuada.

"Yo opino que es muy importante ya que a veces somos un poco ignorantes sobre el tema de la sexualidad, entonces es una opción muy importante que la tomen, no sólo en la carrera de psicología sino en todas las carreras de la universidad. [...] si es muy importante porque a veces como psicólogo te puede llegar un caso o algo que trate sobre sexualidad, entonces pienso que deben de conocer cada punto y por eso creo que es importante." (Miriam)

"Sí, porque como psicólogo que tal si en un futuro te llega un caso de disforia de género, homofobia, violación, todo ese tipo de temas que evidentemente se van a ver en el progreso de sexualidad humana y pues no vas a tener el conocimiento suficiente o vas a saber cómo responder a este tipo de problemáticas, yo creo que es fundamental el tener un conocimiento mínimo de sexualidad o irte preparando". (Jaime)

"Yo opino que debe estar como las obligatorias ya que si vamos a fungir como psicólogos ocupamos como los conocimientos básicos acerca de lo que es la sexualidad humana. Conocer desde tu cuerpo, 
las enfermedades, todo lo referente a la sexualidad humana ya sea que nos lleguen casos referentes a abusos, violaciones y saber cómo actuar ante la situación" (Raúl)

Dos participantes opinaron que no era necesario que la UA fuera obligatoria, sin embargo, indican que sí es importante que se encuentre disponible como UA optativa.

"Yo la considero importante pero no exactamente para la carrera de psicología, sino en general, ser una materia optativa, no tiene que ser obligatoria porque no tiene nada que ver con psicología exactamente". (Elba)

En relación a los contenidos de la UA, hubo un acuerdo en torno al hecho de que, si bien el temario abordado en clase es bastante completo, consideran que es necesario profundizar en torno a algunos temas, como el de la diversidad sexo-genérica.

"Yo creo que sería necesario abordarlo más, menos general y un poco más, para que sea más comprensible, creo que hay muchas cosas que no tocan o falta un poco de diversidad sexual, pero fuera de eso todo estuvo muy bien yo la llevé ya hace casi dos años y estuvo muy bien, pero si me gustaría que fuera un poco más abierto y explícito". (Silvia)

"Sí, considero que sí es adecuado, pero creo importante que profundizaran más en algunos temas ya que a veces se habla como por encimita y quedamos con muchas dudas". (Jaime)

"Me gustó mucho, porque yo ya pasé por esa asignatura, me gustó bastante porque nos adentrábamos a temas, pero había otros temas que dejábamos como, por un lado, como yo no no recuerdo haber visto más a profundidad como disforia de género, identidad y rol, todo ese tipo de cosas que me hubiera gustado visto más a profundidad, lo fuimos viendo, pero conforme la marcha en la universidad, no especialmente en esa clase". (Nadia)

Con respecto a las estrategias didácticas aplicadas en la clase, todos los y las participantes, coincidieron en destacar la importancia de aplicar dinámicas grupales en la clase, así como de llevar a cabo toda clase de actividades que promuevan la participación de los y las estudiantes.

“...durante las clases eran muy dinámicas, las clases nos encargaban materiales, jugábamos con los temas, nos adentramos un poco más a lo que eran los temas, como exposición del maestro tanto como los compañeros, acerca de los métodos anticonceptivos, de las diferentes orientaciones sexuales, a donde acudir, todo lo referente a la sexualidad humana lo mirábamos en la clase. [...] Destacaría las dinámicas, el cómo se manejaba la información, desde la dinámica, trabajo en clase, trabajos de grupo, de quitarle no le quitaría nada, solo en cuestiones de cómo asistir a conferencias, me hubiera gustado asistir a conferencias donde hablarán temas que veíamos en clase donde muchas veces se quedaban en temas de clase, nada más pero no llegábamos a ese punto, pues como temas no, estaba muy completa la clase. "(Raúl)

"Como mencionamos antes, creo que la materia de sexualidad humana es un poco, mm temas que a lo mejor es para unas personas es difícil de abordar entonces en el caso de la materia que llevé se hicieron diferentes actividades hicimos maqueta, participamos todo él salón, había actividades donde no solo éramos equipos de 4 y tenía que pasar uno a exponer, no, sino era todo el grupo que tenía que decir alguna cosa y creo que fue una buena manera de entender los temas y abordarlos al objetivo deseado" (Ana)

"Ahorita que lo mencionaste, yo no me acordaba de esas dinámicas que la maestra hacía, yo me sentía muy cómoda porque no era algo que te exhibía porque todos estábamos participando entonces a mí me gustaba mucho por ejemplo de qué había una pregunta de "¿quién cree que esto es así?" $Y$ te dejaba más enseñanza porque uno tiene esos pensamientos qué, no, teorías de que algo es como

Revista del Laboratorio Iberoamericano para el Estudio Sociohistórico de las Sexualidades https://doi.org/10.46661/relies.5162 
creencias, sin embargo ahí miramos qué no siempre era así, entonces a mí me gustaba mucho que aclararemos eso y era participación de todos no solo de un alumno o de dos". (Silvia)

Seis participantes, además, hicieron hincapié en el hecho de que la exposición de los temas, debe de ser responsabilidad de los y las docentes, ya que ellos son los "expertos"

"...que haya mucha participación de los alumnos, pero también por parte del maestro, es muy importante porque hay veces que los maestros te dejan exponer en toda la clase y ellos no aportan casi nada, entonces pienso que es muy importante de las dos partes que haya participación". (Miriam)

"Yo considero que es más importante que el maestro participe más porque se supone que él es el experto y nosotros estamos ahí para aprender." (Elba)

"De acuerdo con mi compañero también me parece importante lo de las conferencias y otra cosa que se me hace más correcto el hecho de que el tema los imparta el maestro ya que a veces en exposiciones por nuestros compañeros no saben cómo quisiéramos y así podríamos resolver más dudas igual las actividades ayudan mucho a que el aprendizaje quede más concentrado". (Nadia)

Además, cuatro estudiantes se pronunciaron a favor de acudir a conferencias, impartidas por especialistas en el tema de la sexualidad humana.

"Participar abiertamente en el salón o acudir a conferencias porque qué mejor que aprender de un experto o de alguien que ya vivió esa experiencia referente a la sexualidad humana" (Jaime)

En lo referente al tema de las problemáticas que, en materia de salud sexual y reproductiva, enfrenta nuestro país, los y las participantes indican que el principal problema es la falta de información y el desconocimiento que existe en torno a la sexualidad humana, lo cual genera situaciones de descuido a la salud tanto individual como social de las personas. Todos y todas coinciden en que la psicología es una disciplina desde la cual es posible atender estas situaciones a nivel de intervención no solamente individual sino también comunitaria.

"México es un país muy ignorante, en cuestiones de sexualidad, cuestiones referentes al tema de sexualidad, es muy ignorante. La labor como psicólogo es trabajar esa ignorancia, ir a lugares donde desconocen en sí la clase de sexualidad, todo lo que abarca, embarazos no deseados, abortos, qué hacer y qué no hacer con tu cuerpo, violaciones, todo este tipo de cosas que son importantes dentro de la sexualidad humana que él mexicano no comprende. Cuántas veces no hemos visto cosas como transfobia, homofobia, que son aspectos referentes a la sexualidad que él mexicano todavía no acepta desde ver a un hombre vestido de mujer, Como les sigue escandalizando ver a una mujer que decide qué hacer y qué no hacer con su cuerpo, como se escandalizan de ver a una mujer en minifalda él cómo México sigue siendo con él paso de los siglos estando con el paso de los siglos, estando con las mismas reglas retrogradas de siempre, creo que su función principal es educar a las personas". (Raúl)

"Que la mayoría es un poco ignorante o hay ignorancia sobre el tema, como que lo ven muy: 'iAy! es malo', entonces no les gusta o no quieren conocer más sobre el tema". (Miriam)

"Que todavía es tema tabú en este año y pues debería meterse en el sistema educativo estas materias desde antes de la secundaria, pero pues todavía no quieren que los niños no vean esos temas pero es necesario. Esta problemática tal vez se podría resolver desde la psicología con conferencias, talleres, cosas asi." (Elba) 
"Principalmente el problema de México es la ignorancia, es un problema que ha estado desde siempre pero con temas de sexualidad siguen existiendo problemas como homofobia, principalmente problemas para la aceptación, creo que el mexicano sigue siendo como que muy machista o retrógrada en términos de la sexualidad y es eso, el comprender que existe diversidad sexual, que no todo es hombre o mujer que existen personas homosexuales, bisexuales, transexuales, transgénero y que hay que aprender a respetar, creo que es el principal problema de México, el machismo y seguir pensando que debe ser todo conforme a la norma que se estableció entre comillas pero no es así, hay que seguir progresando en temas de sexualidad". (Jaime)

"A mi punto de vista me parece también la desinformación o la mala información que se obtiene, la mayoría de las personas busca información por parte de los amigos o por internet que a lo mejor no es la adecuada y pues recurrente a eso se hace una mala práctica de la sexualidad". (Cecilia)

Llama la atención el hecho de que dos estudiantes, vincularon la desinformación con cuestiones económicas o de clase social,

"Yo creo que también seria ignorancia y pobreza, porque en zonas marginadas las mujeres desconocen mucho sobre los métodos anticonceptivos o pues en sí temas relacionados a la sexualidad en cuanto la cultura, religión, es como que prohíben ese tipo de conocimientos o igual ellas se cierran a obtener esa información". (Diana)

"...en lo que nos compete a nosotros, creo que sería difícil el erradicar la ignorancia, pobreza o tener que ir a cada zona marginal a dar información resulta un poco complicado, pero lo que podemos hacer es a las personas de la comunidad que tenemos cerca, tratar de abrir espacios para que la gente se informe acerca del tema de la sexualidad, el tener conferencistas, el asistir a lugares cercanos a nosotros para llevar temas de sexualidad o temas que actualmente se viven en la sexualidad y que la gente desconoce o normaliza dentro del término de sexualidad como violaciones, comunidad lgbt, cambios de cuerpo, todo ese tipo de cosas que pueden ayudar, el ayudar junto con psicología de la salud, salud comunitaria" (Jaime)

"Que ignoran el tema, personas de escasos recursos o ciertas comunidades que como no tienen la forma de que alguien se los diga o estudiando, no saben qué deben hacer o cómo lo deben hacer, la ignorancia es lo que podría ser y la psicología lo puede resolver dando platicas como lo que te comenté a esas comunidades específicas"

\section{Discusión}

De las 32 instituciones incluidas en el mapeo, 19 cuentan con materias o asignaturas vinculadas con el tema de la sexualidad, de las cuales 15 son obligatorias y 4 optativas. A 9 de estas materias se les ha asignado el nombre de "Sexualidad humana", como en la UABC. El resto de las materias llevan nombres que destacan elementos vinculados con la sexualidad, como son la reproducción, el género, la "Teoría de género", la identidad de género, la afectividad, la "paternidad", la educación, la "coeducación" y la salud. Con base en estas cifras, es posible decir que en las universidades públicas de México, la sexualidad si se considera como un tema necesario en el proceso de formación de los y las profesionales de la psicología. Cabe mencionar que, en el caso de 3 de estas universidades, se cuenta no con una, sino con dos materias vinculadas al tema de la sexualidad en sus programas de licenciatura en psicología.

Con respecto al análisis de contenido realizado al PUA de "Sexualidad humana", aclaramos que se llevó a cabo con la intención de identificar las áreas de oportunidad que nos presenta dicho documento, ya que destacamos el hecho de que dos de los elementos con base en los cuales se 
analizó el contenido del PUA -ODS-ONU (2015) y CCES-UNESCO (2018)-, fueron publicados 3 y 6 años, respectivamente, después de publicado el programa del cual forma parte el PUA analizado.

Si bien en el objetivo del PUA se menciona la "salud sexual integral", así como los aspectos "éticos" y "sociales" de la sexualidad humana, en el resto del PUA, encontramos deficiencias, justamente en estos aspectos. EI PUA se divide en dos unidades, un tema por cada unidad, que a su vez se subdividen en 12 subtemas y 16 sub-subtemas en total. En el sub-subtema 1.1.2 del PUA, se mencionan los "aspectos biológicos, psicológicos y socio-culturales" de la sexualidad y esta es la única mención que se hace en todo el PUA, a los aspectos socio-culturales de la sexualidad, además del sub-subtema 2.7.- "Aspectos éticos y sociales del tratamiento de las disfunciones sexuales", en el cual únicamente se mencionan los aspectos éticos y sociales, pero específicamente vinculados con el tema de las disfunciones sexuales.

Consideramos que no se están tomando en cuenta en el PUA, los siguientes CCES-UNESCO: 2.Valores, derechos, cultura y sexualidad; 3.- Género; 4.- Violencia y seguridad personal; 5.Habilidades para la salud y el bienestar y el subtema 8.2.- Estigma del VIH y del sida, atención médica, tratamiento y apoyo (Ver tabla 3). Lo anterior se considera en virtud de dos cosas: a) Dichos temas no se encuentran mencionados en el PUA y b) Consideramos que es poco probable que se estuvieran considerando para impartirse en el sub-subtema 1.1.2 "aspectos biológicos, psicológicos y socio-culturales" pues son demasiados elementos para revisarse en un solo sub-subtema.

En la UABC existe la UA de "Violencia de género", sin embargo, consideramos que este tema debería de tomarse en cuenta en la UA de "Sexualidad humana" -aunque sea de manera general- pues la UA de "Violencia de género" es optativa y consideramos que el tema de la violencia de género tendría que ser un elemento obligatorio dentro del programa de licenciatura en psicología, además de que esto también podría motivar a los y las estudiantes, a tomar una optativa que les ayude a profundizar en este tema.

Lo anterior se vincula con el hecho de que el PUA no menciona elementos que pudieran vincularse con los siguientes ODS-ONU (Ver tabla 4): 3.7, 5.1, 5.2 y 10.2, los cuales hablan de derechos sexuales y reproductivos, equidad de género, inclusión social, violencia en los ámbitos público y privado y discriminación. El tocar estos temas en el PUA es una necesidad urgente por tres razones: a) Dos de los tres ejes transversales del PDI-UABC son la equidad y la responsabilidad social universitaria b) La inequidad de género, la violencia de género, la discriminación y la falta de educación sexual, son problemáticas que requieren atención urgente en México y c) Los y las psicólogas en formación, perciben la necesidad de atender a las problemáticas anteriormente mencionadas en la comunidad.

Llama la atención que la mitad del PUA está dedicado al tema de las disfunciones sexuales y que se incluye el sub tema 2.6.- "Esquema de atención para no especialistas". Lo anterior debido a que el artículo 30 del código ético del psicólogo, indica que: "El psicólogo efectúa solamente las intervenciones para las cuales posee la educación, formación o experiencia supervisada, y la pericia necesarias" (SOMEPSO, 2007: 54). Consideramos que: a) Una parte de un PUA no constituye formación suficiente para llevar a cabo una intervención sexoterapéutica b) La atención terapéutica en materia de disfunciones sexuales, exceden las facultades de un o una licenciada en psicología y c) La enunciación de este sub-subtema, sugiere que es posible llevar a cabo intervenciones sexoterapéuticas, siendo una persona "no especialista", lo cual nos parece contrario a lo que recomienda el código ético del psicólogo. 
De los 11 elementos que se encuentran apuntados como referencias bibliográficas en el PUA, tres son libros, dos son manuales -uno de ellos de metodología y otro, vinculado con la UA de "Sexualidad humana"-, uno es un libro de teorías de la personalidad y los restante ocho, son libros de sexualidad, amor y erotismo, de los cuales, el más reciente fue publicado en el 2004. Además, se recomienda la película "Éxtasis", como recurso didáctico. Si bien, en el PUA, no es posible encontrar más datos de la película que se sugiere, asumimos que se trata de la película "Éxtasis" de Gustav Machaty, estrenada en 1934 y que, en aquella época, fue considerada como transgresora, pues mostraba desnudos completos y la escena de un orgasmo femenino.

En los grupos focales, se encontró que los y las estudiantes consideran que la UA de "Sexualidad humana" debería de estar disponible para todas las carreras, no solamente para psicología. Lo anterior debido a la educación sexual deficiente recibida en niveles educativos anteriores. Cabe mencionar que aunque la UA de "Sexualidad humana" se encuentra disponible para cualquier estudiante que desee cursarla -previa autorización por parte de su tutor o tutora académica- este señalamiento pudiera apuntar a la necesidad de espacios de formación integral y orientación en sexualidad, a disposición de los y las universitarias, más allá de las UA de los programas.

Con respecto a los contenidos del PUA, se indicó la necesidad de profundizar en el tema de la diversidad sexo-genérica. Con respecto a este punto, el sub-subtema 1.1.4 del PUA, hace mención de la "Homosexualidad, bisexualidad y heterosexualidad", si tomamos en cuenta los significados de las siglas LGBTTTIQA (lésbico-gay-bisexual-transexual-travesti-transgénero-intersexual-queerasexual) podemos decir que los tres elementos mencionados en el sub-subtema 1.1.4, resultan insuficientes para revisar el tema de la diversidad sexo-genérica.

Los y las estudiantes indicaron que, si bien es necesario llevar a cabo dinámicas y actividades varias que requieran de la participación de todo el grupo, la responsabilidad de explicar los temas, tendría que recaer en el o la docente. También señalaron la importancia de participar en conferencias adicionales a la clase, para complementar sus conocimientos.

Con respecto a la dinámica de clase, los y las estudiantes hablaron de la utilidad de abordar la sexualidad con apertura, pero sin llevar a cabo actividades que recurrieran a "exhibir" a los y las participantes, ya que si bien es necesario romper los tabúes que se han construido en torno al tema de la sexualidad, es indispensable mantener interacciones respetuosas que apunten a discutir en torno a las creencias construidas colectivamente, a los hechos sociales, a los datos aportados por la ciencia y a las posibilidades que la profesión nos ofrece para intervenir de manera ética y responsable en nuestras comunidades. La clase de sexualidad no es una "exhibición" de intimidades, sino una construcción de argumentos profesionales.

Los resultados de los grupos focales, son similares a los encontrados por Moral y Ortega (2009), no así a los encontrados por otros autores (Chávez, Petrzelová y Zapata, 2009; Cañizo y Salinas, 2010 y Hernández et al., 2017) ya que los estudiantes reconocen la necesidad que existe de respetar e incluir socialmente a los miembros de la comunidad LGBT+, lo cual coincide con lo encontrado en la ENADIS, realizada por CONAPRED (2010).

Sin embargo, los estudiantes no hicieron mención de la violencia de género y del abuso sexual infantil como problemáticas urgentes a resolver mediante la educación integral de la sexualidad, lo cual nos lleva a pensar que es probable que estos fenómenos, detectados en estudios como aquellos llevado a cabo por el INEGI (2016) y la OCDE (Senado de la República, 2019), se encuentren invisibilizados entre los estudiantes. En una de las declaraciones, se identificó como un problema el 
hecho de que "las mujeres" no tuvieran información acerca de los métodos anticonceptivos. Esta declaración está vinculada a la creencia de que la reproducción es una responsabilidad de las mujeres exclusivamente.

Llama la atención el hecho de que la desinformación o "ignorancia" en torno al tema de la sexualidad humana se vincula con la "pobreza" o con la gente de "zonas marginadas", lo cual nos indica que se está invisibilizando el hecho de que esta desinformación en materia de sexualidad, se presenta en todos los estratos sociales, algo que nos afecta profundamente como sociedad. Los y las participantes coinciden en señalar que el dar solución a la desinformación en sexualidad y a muchas problemáticas vinculadas con ella, es algo que es parte del campo de acción de los y las profesionales de la psicología. Lo anterior apoya lo dicho por Álvarez-Gayou (1998) quien habla de la importancia de contar con un entrenamiento profesional en materia de sexualidad humana, que tenga el objetivo de promover la salud sexual integral de las comunidades.

Los hallazgos también apuntan al abordaje de la sexualidad como un tema que trasciende el plano meramente biológico y toca tanto lo psicológico, como lo emocional, lo social y lo cultural, lo cual se vincula con la definición de sexualidad que hace la UNESCO (2014) y con lo dicho por autores como Álvarez-Gayou (1998) y Rosales-Mendoza y Salinas-Quiroz (2017) quienes abogan por un abordaje integral del tema de la sexualidad humana.

\section{Conclusiones y recomendaciones}

La inclusión de la UA de "Sexualidad humana" en el programa de licenciatura en psicología de la $U A B C$ es un primer paso hacia el proceso de formar de manera integral en sexualidad a los y las estudiantes. Sin embargo, la evaluación, tendría que ser una acción que acompañe de manera constante este esfuerzo. De esta primera aproximación, concluimos que:

a) La sexualidad humana es un tema reconocido como necesario en la formación en psicología, por las instituciones de educación superior públicas -incluida la UABC- del país, por autores y autoras que han estudiado el tema -citados en este documento-y por los y las estudiantes incluidas en este estudio.

b) Es necesario incluir en el PUA de "Sexualidad humana" de la UABC, temas que atiendan a la dimensión socio-cultural de la sexualidad, como son: valores y sexualidad; cultura y sexualidad; derechos sexuales y reproductivos; violencia de género; inclusión y diversidad sexual y estigma e ITS.

c) Los temas anteriormente mencionados, tendrían que vincularse con los ODS-ONU y los ejes transversales de equidad y responsabilidad social del PDI-UABC, de tal manera que los aspectos socio-culturales de la sexualidad, sean retomados desde una perspectiva política, apuntando a la construcción de ciudadanía sexual, en términos de salud, inclusión y bienestar comunitario.

d) La clase de "Sexualidad humana" tendría que incluir dinámicas y actividades grupales, así como participación en cursos, talleres y conferencias externos a la clase.

e) EI PUA tendría que incluir, en su sección de bibliografía, libros directamente relacionados con la sexualidad humana, que se encuentren actualizados, así como artículos académicos actuales, contextualizados en México y América Latina, principalmente. Asimismo, 
sugerimos la inclusión de documentales, en vez de películas, ya que estas últimas se generan con el objetivo de entretener, no precisamente de informar o formar.

f) Se recomienda generar espacios al alcance de toda la comunidad universitaria, en los que se informe y se oriente en materia de sexualidad humana, de una manera objetiva, integral e inclusiva, con el objetivo de contribuir al desarrollo personal de los y las estudiantes y de promover la formación integral de estudiantes cuyas carreras se encuentren vinculadas con la salud, la educación y el aspecto social del ser humano. 


\section{Bibliografía}

Álvarez-Gayou, J.L. (1998). La educación profesional de la sexualidad: una necesidad impostergable en México, Vol. I. México: UNAM.

Cañizo, E. y F. Salinas (2010). Conductas sexuales alternas y permisividad en jóvenes universitarios. Enseñanza e Investigación en Psicología, 15 (2), 285-309. Disponible en:

https://www.redalyc.org/articulo.oa?id=292/29215980004

Chávez, M., Petrzelová, J. y J. Zapata. (2009). Actitudes respecto a la sexualidad en estudiantes universitarios. Enseñanza e Investigación en Psicología, 14 (1), 137-151. Disponible en:

https://www.redalyc.org/articulo.oa?id=292/29214110

Consejo Nacional de Población (CONAPO) (2017). Situación de la salud sexual y reproductiva. Entidades federativas. Baja California. Disponible en:

https://www.gob.mx/conapo/documentos/sitacion-sexual-y-reproductiva-de-las-entidades-

federativas?idiom $=\mathrm{es}$

Consejo Nacional para Prevenir la Discriminación (CONAPRED) (2010). Encuesta Nacional sobre Discriminación en México. Resultados sobre Diversidad Sexual. Disponible en:

http://www.conapred.org.mx/userfiles/files/Enadis-2010-DS-Accss-001.pdf

González, Y. (2015). Significados acerca de la sexualidad en estudiantes de psicología en Colombia, Revista Latinoamericana Sexualidad, salud y sociedad, 136-153. Disponible:

http://www.scielo.br/pdf/sess/n21/1984-6487-sess-21-00136.pdf

Hernández, Y., Peláez, F. E., Serrano, A.C., Barros, S. M., Cedillo, M.L. y O. S. Vanegas (2017). Creencias, actitudes y prácticas en sexualidad en estudiantes de psicología clínica de la universidad católica de Cuenca, en Revista Electrónica de Psicología Iztacala, 20 (3), 307-336. Disponible en: https://www.iztacala.unam.mx/carreras/psicologia/psiclin/vol20num3_monografico/Vol20No3Art 17.pdf

Instituto Nacional de Estadística y Geografía (INEGI) (2016). Encuesta Nacional sobre la Dinámica de las Relaciones en los Hogares. Disponible en:

https://www.inegi.org.mx/contenidos/programas/endireh/2016/doc/endireh2016_presentacion_ ejecutiva.pdf

Moral, J.M. y E. Ortega (2009). Representación social de la sexualidad y actitudes en estudiantes universitarios mexicanos, en Revista de Psicología Social, 24 (1), 65-79. Disponible en:

https://www.researchgate.net/publication/233630533_Social_representation_of_sexuality_and_a ttitudes_in_Mexican_university_students

Organización de las Naciones Unidas (ONU) (2015). Objetivos de desarrollo sostenible. Disponible en:

https://www.un.org/sustainabledevelopment/es/objetivos-de-desarrollo-sostenible/

Organización de las Naciones Unidas para la Educación, la Ciencia y la Cultura (UNESCO) (2014). Educación integral de la sexualidad: conceptos, enfoques y competencias. Chile: UNESCO.

http://unesdoc.unesco.org/images/0023/002328/232800S.pdf

Revista del Laboratorio Iberoamericano para el Estudio Sociohistórico de las Sexualidades https://doi.org/10.46661/relies.5162 
Organización de las Naciones Unidas para la Educación, la Ciencia y la Cultura (UNESCO) (2018). Orientaciones técnicas internacionales sobre educación en sexualidad. Disponible en:

https://www.who.int/reproductivehealth/publications/technical-guidance-sexuality-education/es/

Organización para la Cooperación y el Desarrollo Económico (OCDE) (2020). Over the rainbow? The road to $L G B T I$ inclusion. Disponible en:

https://www.oecd.org/mexico/OECD-LGBTI-2020-Over-The-Rainbow-MEXICO.pdf

Rosales-Mendoza, A. y F. Salinas-Quiroz (2017). Educación sexual y género en primarias mexicanas ¿qué dicen los libros de texto y el profesorado? Revista Electrónica Educare, 21 (2), 1-21

http://dx.doi.org/10.15359/ree.21-2.11

Senado de la República (2019). Boletín del 24 de agosto de 2019. Disponible en:

http://comunicacion.senado.gob.mx/index.php/informacion/boletines/45796-mexico-primer-

lugar-en-abuso-sexual-infantil.html

Sociedad Mexicana de Psicología (SOMEPSO) (2007). Código ético del psicólogo. Trillas.

Universidad Autónoma de Baja California (UABC) (2012) Plan de estudios del programa de licenciado en psicología.

Universidad Autónoma de Baja California (UABC) (2019) Plan de Desarrollo Institucional 2019-2023. Disponible en:

http://pedagogia.mxl.uabc.mx/transparencia/PDI/PDI_UABC_2019-2023.pdf 